\title{
Industrial diagnostics system using gamma radiation
}

\author{
Adrian Jakowiuk, \\ Eukasz Modzelewski, \\ Jan Pieńkos, \\ Ewa Kowalska
}

\begin{abstract}
During the operation of large industrial installations, a very important task is to maintain the proper technical state. In the event of an emergency, it is vital to locate the place of occurrence as soon as possible. In solving this type of problem, it often helps to apply the methods of measurement associated with ionizing radiation. One of these methods is the gamma scanning. The purpose of this type of measurement is the detection and localization of disturbance of technological processes which may result in incorrect decomposition the flowing medium and workpiece (sediments, congestion) as well as damage to the internal constructions. A particularly: (i) preventive diagnosis - early detection of installation failure; (ii) rationalization of repairs and renovations - to determine the need to take or not to take remedial action; (iii) quick and precise installation inspections - to gain knowledge of the technical condition and technological installations; (iv) indication of worn parts and posing a threat - diagnostics of the technical condition installation; (v) forecasting the useful lifetime of equipment.
\end{abstract}

Key words: diagnostic system $\bullet$ gamma radiation

\footnotetext{
A. Jakowiuk ${ }^{\bowtie}$, 七. Modzelewski, J. Pieńkos, E. Kowalska Institute of Nuclear Chemistry and Technology,

16 Dorodna Str., 03-195 Warsaw, Poland,

E-mail: ior@jakowiuk.pl
}

Received: 29 September 2014

Accepted: 20 May 2015

\section{Introduction}

During the operation of large industrial installations, a very important task is to maintain their proper conditions. In the event of a state of emergency it is also important to locate the place of malfunctioning as soon as possible. This often is associated with the necessity of interrupting the production process and the need to intervene in the structure of the installation by its disassembly [1]. This solution entails considerable financial losses. Expedient, thus becomes a search and use of such diagnostic methods that do not require interruption of the technological process and in real time may provide information about the degree of wear (or pollution) of the tested elements of the installation. The measurement methods associated with the use of ionizing radiation helps in an embodiment of this type of problems [2].

\section{Purpose of a diagnostic system}

In the petrochemical industry, regularity of the technological process and the reliability of the installation play a very important role. There is a need for preventive maintenance, replacement of damaged parts, and other measures to prevent the occurrence of unexpected downtime. This increases the usefulness of the installation and therefore minimizing production costs. 
The solution to the problem posed above is the use of ionizing radiation. This makes it possible to install the test gamma radiation (gamma scanning) at different cross sections [3] and on the basis of obtained values locate the place and type of damage. The main problems associated with the development of such a diagnostic system were: the development of the gamma scanning equipment to enable overexposure of installations in various systems of geometry source - probe and development of appropriate mathematical models that allow prediction of the degree of the object degradation on the basis of single and periodic scans of the tested installation.

As a result of the undertaken work, the diagnostic system used in large industrial installation created gamma radiation. By using multivariate statistical analysis and trend analysis, both the assessment and forecast of the technical condition of the tested installation can be made. The use of apparatus which allows performing layered of gamma radiation in different geometries and adaptation of algorithms used in image reconstruction in computed tomography allows for more detailed localization of disturbance in the studied processes.

\section{Gamma Scanner - principle of operation}

Principle of operation of the scanner illustrates the functional diagram shown in Fig. 1. Container Z contains the gamma radiation source, scintillation probe (SS), as a gamma radiation detector, which is moved simultaneously up and down using the drive source (NZ), and drive of the probe (NS). Scanning takes place under the control of a portable PC (industrial computer) equipped with appropriate software.

During the test, the source of radiation and scintillation detector probe are moved every few centimeters, and the measurement of the speed of counting impulses is done. On the basis of the measurements of the speed of counting impulses, the average density is calculated and the graph of density in the function of height of the tested installation is obtained.

\section{Industrial installations}

Operation of designed system has been tested on two different installations. The first of these was

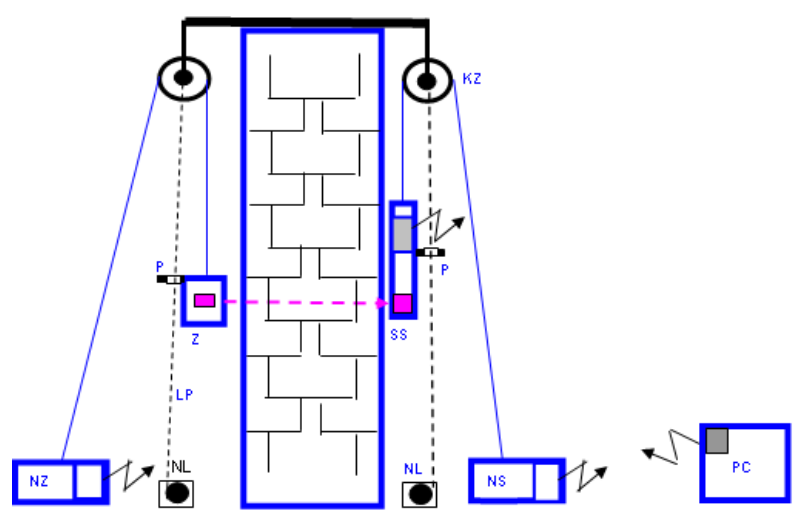

Fig. 1. Functional diagram of Gamma Scanner.

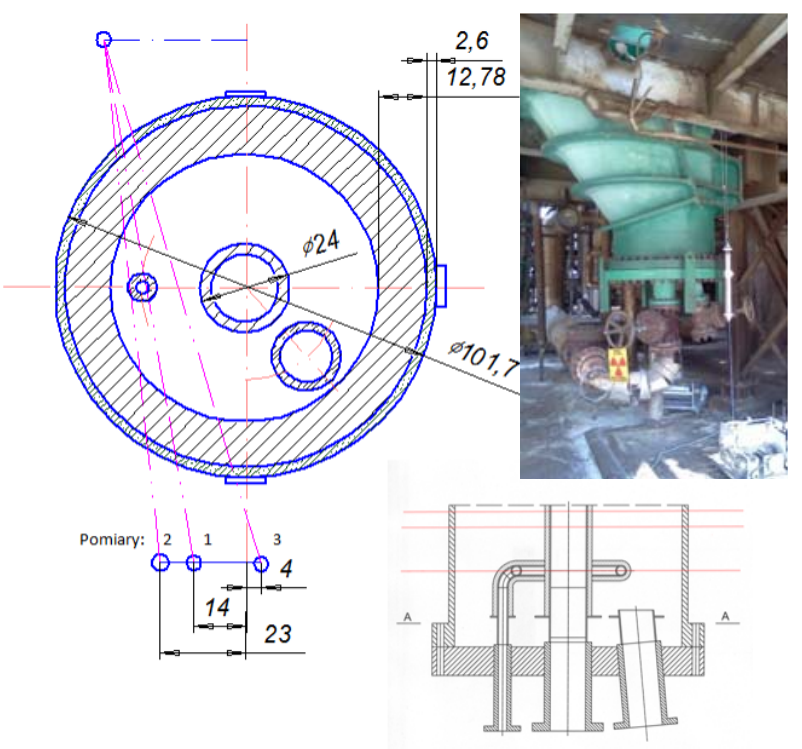

Fig. 2. Diagram of tested riser pipe with measurement geometry. (Source - on the upper part of the figure, the three probes locations - on the bottom of the drawing).

the installation of riser pipe to catalytic cracking and the second was the laboratory installation for obtaining the biogas.

\section{Installation of catalytic cracking}

Preliminary studies of Gamma Scanner were conducted on installation of riser pipe to catalytic cracking (Fig. 2). In 2013, three series of measurements were made; their aims were to explore the potential of measuring equipment and to familiarize with the tested installation. During the fourth session of the gamma radiation measurement, the installation was tested in three cross sections. The scanning results are shown in Fig. 3. Obtained graphs were compared with possessed technical documentation, and on this basis, places where the results deviate from the expected were selected. They are indicated on the graph in red. The most likely place where coke can be deposited is at a place of height about 85 to $90 \mathrm{~cm}$.

\section{Laboratory installation for obtaining biogas}

The Laboratory of Nuclear Control Systems and Methods (INCT, Warsaw) carry out work related to obtaining biogas. The important parameters describing the process are the level of liquid phase inside the bioreactor (fermentor) and information concerning the presence of foam arising during the process.

Initial installation tests were performed using two sources of radiation ${ }^{137} \mathrm{Cs}$ and ${ }^{60} \mathrm{Co}$. Source and probe were at a distance of $90 \mathrm{~cm}$. A photograph of the tested installation with the measuring system is shown in Fig. 4. Installation was scanned in its middle part. For each source, three measurements were performed. The obtained results are shown in Fig. 4. 


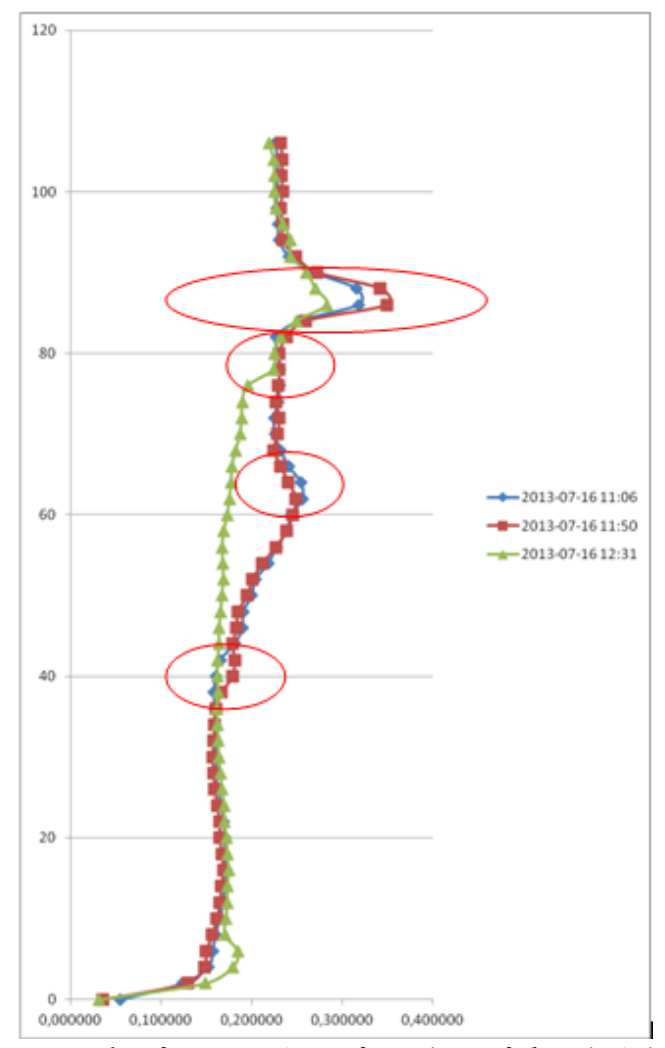

Fig. 3. Result of X-rays (as a function of density) in the three cross sections of the installation of riser pipe to catalytic cracking. Ellipses mark areas that deviate from expected results on the basis of its technical documentation.

\section{Methods for the analysis of the measurement results}

The arising diagnostic system (Fig. 5) provides the use of selected methods in the field of multivariate statistical analysis and time series analysis. These are:

- Principal component analysis (PCA) [4]. It will be used to reduce the measurement uncertainty resulting from the method of measurement (registration gamma radiation). This method should also allow the separation of the solid component (related to the shape of the object). This allows transmittal of the signal imaging the changes in the tested installation for further processing. Examples of the results of analysis for the simulated data are shown in Fig. 6.

- Partial least squares (PLS) [5]. It is expected that the application of this analysis allows the assessment of the degree of degradation (the changes) in the tested object. This will be possible only in the case of installations for which degree of wear will be known (e.g., deposition of coke), degrees that can be referred to the obtained scan results.

- Trend analysis. The use of regression analysis should allow the prediction of the future state of the object. To evaluate the future state of the tested installation, the basic methods associated with the analysis of the trend will be used.

- Image reconstruction [6]. The measurement methods used in computer tomography (CT) allow to obtain images of the internal contents
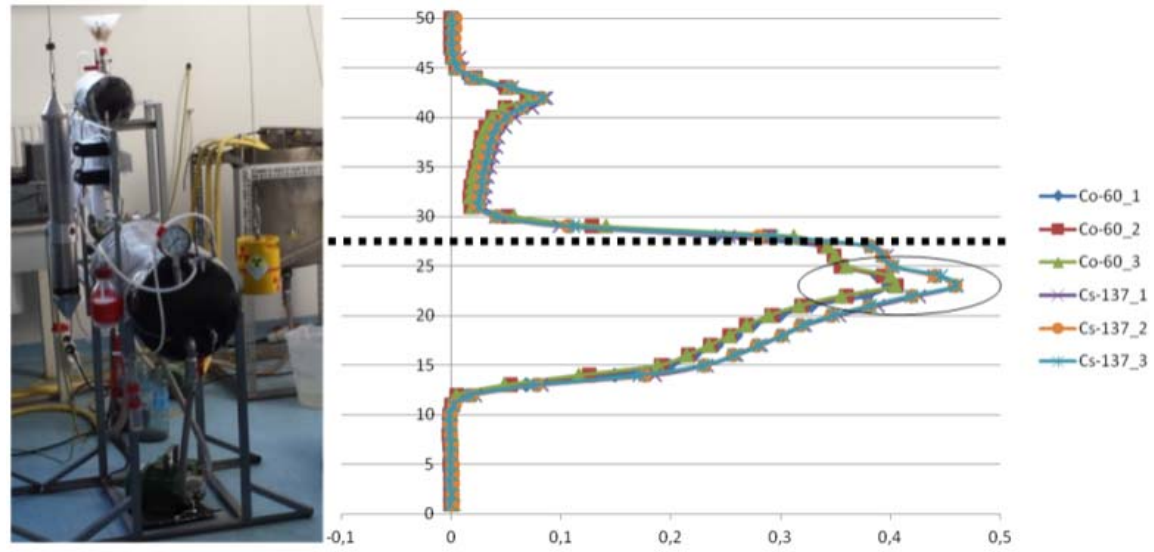

Fig. 4. Installation for obtaining biogas with a visible measurement system. The graph shows the results of the scan as a function of density.

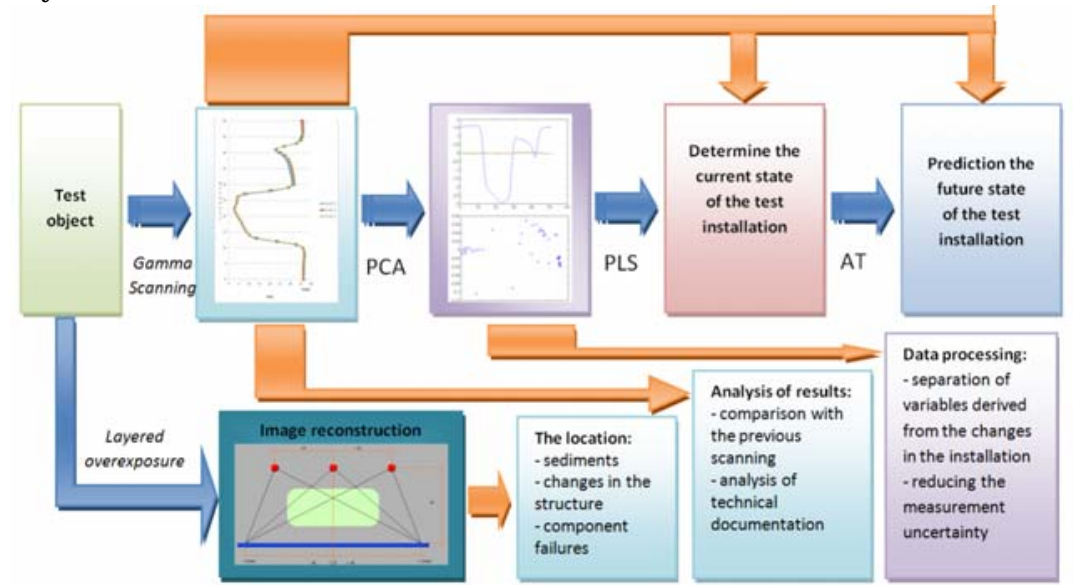

Fig. 5. Functional diagram of the diagnostic system. 


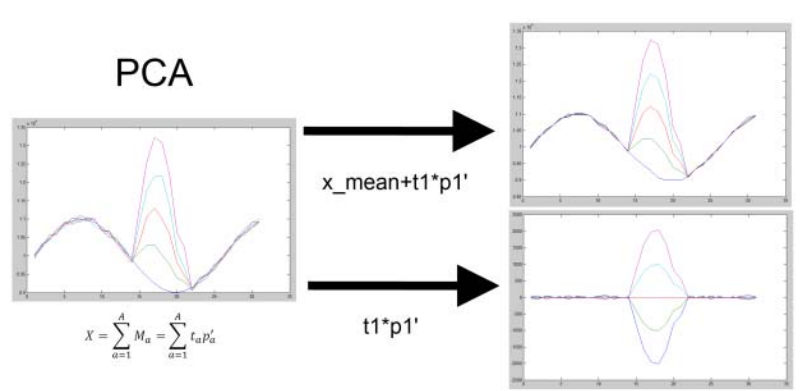

Fig. 6. PCA analysis result for the simulated input data.

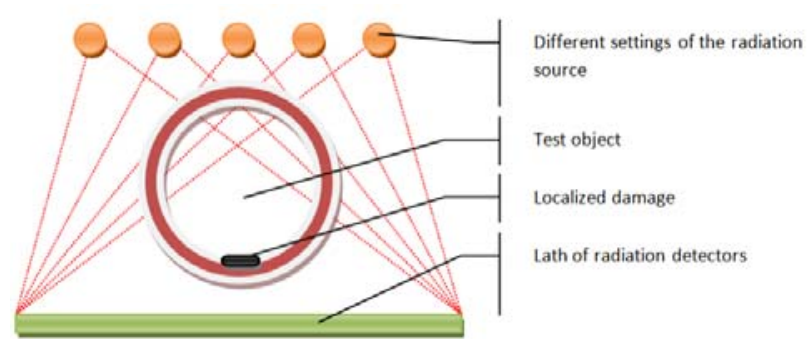

Fig. 7. The proposed geometry measurement for X-rays of layered industrial installations.

of the tested installation. However, in many industrial plants, it is not possible to measure the geometry of such a setting that can be applied to reconstruct the overexposed layer of known algorithms used in CT. Frequently, it is possible to apply, in its various configurations, the geom- etry shown in Fig. 7, which leads to the need of developing new algorithms taking into account the measurements at a specific orientation of the source - probe.

These algorithms will be checked on the tested laboratory installation and then on the medium-laboratory installation for obtaining biogas located at the INCT area, and their usefulness in industrial applications will be tested on the target industrial installation.

\section{References}

1. Mihułek, M. (Ed.). (2003). Charakterystyka technologiczna rafinerii ropy $i$ gazu w Unii Europejskiej. Warsaw: Ministerstwo Środowiska.

2. Rada do Spraw Atomistyki. (2006). Strategia rozwoju atomistyki w Polsce. Warsaw: Państwowa Agencja Atomistyki.

3. Machaj, B., Jakowiuk, A., Świstowski, E., \& Palige, J. (2011). Operation manual of Gamma Scanner. Warsaw: Institute of Nuclear Chemistry and Technology.

4. Krzanowski, W. J. (2000). Principles of multivariate analysis: A user's perspective. Oxford University Press.

5. Rencher, A. C. (1997). Multivariate statistical inference and applications. Department of Statistics, Brigham Young University.

6. Kak, A. C., \& Slaney, M. (1988). Principles of computerized tomographic imaging. New York: The Institute of Electrical and Electronics Engineers, Inc. 